\title{
Sensor Fault Detection in Etch Based on Broadband RF Signal Observation
}

\author{
H.-M. Park*, and J. W. Grizzle \\ Electronics Manufacturing and Control Systems Laboratory, \\ Dept. of Electrical Engineering and Computer Science, University of Michigan \\ Ann Arbor, MI 48109-2122, USA
}

\begin{abstract}
To minimized the machine down time in semiconductor manufacturing process, a novel sensor fault detection in a plasma etching system employing a Broadband RF sensor was developed. The work presented here focuses on detecting errors in the TCP measurement and pressure sensor, though the method has potential for several of the other sensors. To improve the detection sensitivity, we used real-time machine input estimation, and compared it from actual recipe setting instead of employing raw RF signals directly. For a main etch process on a Lam TCP 9400SE, $\pm 10 \%$ relative deviations in TCP measurement, or $\pm 25 \%$ in pressure simulating respective TCP and pressure sensor faults in etching process can be detected with high accuracy: Detection probability $P_{D} \approx 1$ and false alarm rate $P_{F} \approx 0$ for both.
\end{abstract}

*Author to whom correspondence should be addressed. Tel: 734-764-4321, Fax: 734-763-8041, email: parkhm@eecs.umich.edu 


\section{INTRODUCTION}

As described by Bogardus [1], fault detection studies in semiconductor manufacturing are motivated by the needs to reduce 1) equipment downtime by rapid identification and characterization of a fault, 2) the number of scrap wafers by identifying when the equipment is not operating properly, and 3) the number of test wafers by identifying optimum equipment operating conditions. Malfunction should be avoided by means of preventive maintenance or, at least, be detected and recovered from as soon as possible to minimize machine downtime and loss of wafers. Machine malfunction caused by mechanical subsystem failure, such as a broken wafer transfer robot arm, is easy to detect via visual inspection. However, sensor errors caused by sensor aging, miscalibration, or drift are insidious and cannot be easily detected until scheduled maintenance or post-process wafer quality check. With the decrease in device feature size and increase in wafer size, a tight control of the process is critical. This requires tighter process error and drift margin. Thus, even slight errors in sensors can significantly impact the process result. To reduce COO, errors in sensors should be detected in real time and corrected as soon as possible.

Various fault detection and classification techniques have been applied in semiconductor manufacturing areas: photolithography [2], Plasma Enhanced Chemical Vapor Deposition (PECVD) [3], Low Pressure Chemical Vapor Deposition (LPCVD) [4], and plasma etch [5-9]. For instance, equipment models to diagnose equipment malfunctions at a given process step were developed and applied to detect faults in the PECVD nitride process [3]. For the identification of gas flow controller malfunctions in the Reactive Ion Etching (RIE) system, neural network trained on the monitored data using a feed-forward, error back-propagation algorithm was used [7,9]. An expert system that automatically interprets the traces was also designed and tested on the plasma etch process [5].

In this paper, we propose a new technique of sensor fault detection based on Broadband Radio Frequency (BRF) sensor signal observation. Specifically, we applied this RF sensor based fault detection to the RIE system to identify process abnormality caused by sensor fault in real time. By observing the broad range of plasma impedance variation, the Broadband RF sensor enables us to pinpoint the exact source of the abnormality. For fault detection, the etching process was selected since it is a fundamental and irreversible step in chip production. Therefore, accurate and timely detection of sensor faults in this area is critical to the reduction of production cost. We also employed a non-parametric sign test to decide the occurrence of sensor fault via Broadband RF sensor signal observation. The non-parametric detection method is a useful and effective method when the probability density func- 
tion (pdf) of the detector input is unknown [10]. The non-parametric detection method provides stable performance regardless of the exact pdf.

We utilized the Broadband RF sensor's unique characteristic to detect process abnormalities caused by sensor fault in real time, and furthermore, pinpoint the source of the abnormality by observing the broad range of plasma impedance. Plasma impedance contains important information about plasma characteristics such as ion concentration (resistance) and sheath thickness (reactance), which are factors critical to the etch rate and etch profile. The plasma impedance is strongly dependent on the specific plasma generation parameters such as pressure, supplied power, gas flow rate, and chamber conditions. A number of researchers have investigated plasma impedance. Maynard et al. used RF metrology for the endpoint detection of the etching process based on the change of impedance when a new film layer was exposed to the plasma [11]. Scanlan applied the plasma impedance and ion flux monitoring to study plasma drift and ion flux uniformity [12]. Garvin at the University of Michigan pursued a new Broadband RF sensing technique [13]. The conventional RF sensing technique only measures the impedance of the fundamental frequency (typically $13.56 \mathrm{MHz}$ ), which is the frequency of the Alternating Current (AC) power supply used to generate the plasma, or couples of harmonic frequencies at most [14]. The distinctive feature of Garvin's Broadband RF sensing technique includes that it scans a wide frequency range, typically $1 \sim 2 \mathrm{GHz}$, and analyzes the collected RF signal as a whole. Thus, a small variation of plasma conditions can be detected, which is impossible by conventional RF sensor measurement. Figure 1 illustrates the comparison of the reflectance coefficient in magnitude vs. frequency between an empty chamber (no process) and a chamber under the poly-Si Main Etch (ME). Two resonant peaks appear in the range of $1 \sim 1.5 \mathrm{GHz}$ under the ME of poly-Si whereas rather noisy peaks in $2 \sim 2.5 \mathrm{GHz}$.

In the sensor fault detection experiment, we assume the following conditions:

A. The RF sensor is more reliable and robust than the other sensors in the plasma etching system.

B. Only one sensor can fail at a time.

C. Sensor abnormality can be simulated by a change in the machine input setting.

Its simple configuration and lack of moving parts make the Broadband RF sensor more robust compared to the other sensors in the plasma etching system. Because the probability of simultaneous occurrence of faults in two or more sensors is extremely low, we have chosen to ignore this scenario as an experimental condition. Moreover, instead of miscalibrating or tampering with the sensor, we intentionally changed the machine input setting to create sensor fault. For instance, if the pressure 
sensor is malfunctioning, then the actual chamber pressure would be different from the nominal pressure due to the . Hence, we changed the pressure setting from its nominal value to simulate a pressure sensor fault.

Section II reviews the fundamentals of detection theory, and then Section III is followed by a brief description of the experimental setup. The Design of Experiment (DOE) to extract the distinctive RF sensor signal fingerprints under the sensor fault occurrence is explained in Section IV. Section V discusses the experimental results of the TCP and pressure sensor fault detection system. We conclude with a summary in Section VI.

\section{Detection Background}

A typical detection system operates on binary decision making. Once a detection signal is observed, one of two decisions must be made, typically: yes/no, accept/reject, or 0/1. In detection theory, we label the two possible choices null hypothesis $\left(H_{0}\right)$ and alternative hypothesis $\left(H_{1}\right)$. When the detector input is a random signal, $z$, the output is a random variable, $D(z)$, which depends on the input $z$ (Fig. 2). The random variable $D(z)$ has the value 0 or $1, H_{0}$ or $H_{1}$, respectively. If the set of hypotheses is limited to two, the problem is a binary hypothesis testing problem. However, if there are $\mathrm{M}$ hypotheses with $\mathrm{M}>2$, the problem becomes a multiple-hypothesis testing or $M$-ary detection problem. Detector performance can be evaluated by means of two different types of detector error: type I, false alarm, and type II, miss. False alarm is deciding $H_{1}$ when $H_{0}$ is true. The probability of false alarm $\left(P_{F}\right)$ is:

$$
P_{I}=P_{F}=P\left(D_{1} \mid H_{0}\right)=\int_{z_{1}} p\left(z \mid H_{0}\right) d z .
$$

On the other hand, miss is deciding $H_{0}$ when $H_{1}$ is true. And, the probability of miss $\left(P_{M}\right)$ is:

$$
P_{I I}=P_{M}=P\left(D_{0} \mid H_{1}\right)=\int_{z_{0}} p\left(z \mid H_{1}\right) d z .
$$

Similarly, the probability of detection $\left(P_{D}\right)$, which is a more general measure of detector performance, is given by:

$$
P_{D}=P\left(D_{1} \mid H_{1}\right)=\int_{z_{1}} p\left(z \mid H_{1}\right) d z=1-P_{M}
$$

Ideally, $P_{D}$ and $P_{F}$ should be 1 and 0 , respectively. Plotting the detection probability $\left(P_{D}\right)$ against the false alarm probability $\left(P_{F}\right)$, known as Receiver Operating Characteristics (ROC), is useful for describing the performance of a detector. 
Detection methods can be categorized as either parametric or non-parametric detection [10]. Parametric detection assumes that the conditional probability density functions, $p\left(z \mid H_{0}\right)$ and $p\left(z \mid H_{1}\right)$, are known. These probability density functions are then used to arrive at a detector $D$. The most general form of parametric detection is Bayes' criterion. Given the cost for each decision, the decision regions $Z_{0}$ and $Z_{1}$ are selected so that the expected value of cost, $E($ cost $)=E\left(\right.$ cost $\left.\mid H_{0}\right) P\left(H_{0}\right)+E\left(\operatorname{cost} \mid H_{1}\right) P\left(H_{1}\right)$, is minimized. In short, the decision is expressed as:

$$
\frac{p\left(z \mid H_{1}\right)}{p\left(z \mid H_{0}\right)} \underset{H_{0}}{\stackrel{H_{1}}{\gtrless}} \frac{P\left(H_{0}\right)\left(C_{10}-C_{00}\right)}{P\left(H_{1}\right)\left(C_{01}-C_{11}\right)},
$$

where $C_{i j}$ is the cost associated with making decision $D_{i}$ when the true hypothesis is $H_{j}$. When the a priori probabilities, $P\left(H_{0}\right)$ and $P\left(H_{1}\right)$, and each cost function are unknown, the Neyman-Pearson decision criterion can be used. This method is named the most powerful $(M P)$ test since it achieves the largest $P_{D}$ among all the tests that have the same type I error probability $\left(P_{F}\right)$. Nonetheless, these parametric decision criteria require the input probability density function (pdf), typically, in the form of a Gaussian or an exponential distribution to facilitate analysis. However, in many cases, the form of input probability density function is not given or difficult to define. On the other hand, nonparametric methods, also called distribution-free methods, do not assume that the input probability density functions are completely known, but only make general assumptions about the detector input such as symmetry of the probability density function and continuity of the cumulative distribution function. Since parametric detectors are based on specified forms of the input probability density functions, their performance may vary widely depending on the actual density function of the input $z$. Non-parametric detectors, however, maintain a fairly constant level of performance because they are based on general assumptions of the input probability density. The simplest non-parametric detector, called a sign test, bases its decision solely on the signs of the input observations. This detector has the for

$$
D(x)=\left\{\begin{array}{lll}
0 & \text { if } & \sum_{i=1}^{n} u\left(z_{i}\right)<T \\
1 & \text { if } & \sum_{i=1}^{n} u\left(z_{i}\right)>T
\end{array}\right.
$$

where $u\left(z_{i}\right)$ is the unit step function, $\sum_{i=1}^{n} u\left(z_{i}\right)$ is known as the test statistic, and $T$ is the threshold of the test. Due to the difficulty of defining the probability density function of the detector input, we applied this non-parametric method to the sensor fault detection in this study. 


\section{EXPERIMENTAL SETUP}

\section{A. Hardware}

\section{A.1 The Plasma Etching System: Lam TCP 9400SE}

For the etch of a deep sub-micron patterned wafer, a low-pressure, high-plasma density Lam TCP 9400SE etcher was used (Fig. 3). As a dual-power-source etcher, the TCP 9400SE employs a "transformer coupled" RF power supply to set plasma density and a standard capacitively coupled RF "bias" power supply to set ion energy. To maximize the delivered power to the plasma reactor and protect the power supplies and cables, a matching network is connected to the power source and electrode. The etcher is equipped with a six-inch bipolar electrostatic chuck with helium backside cooling. The temperature controller of the bottom electrode was set to $60 \mathrm{C}$ and chamber temperature to $50 \mathrm{C}$ throughout the experiment. This plasma etcher is designed for six-inch or eight-inch wafers with subhalf-micron pattern etch of poly-Si, refractory metal silicides, organic anti-reflection coating (ARC), nitride, and dry photoresist, and thus, $\mathrm{C}_{2} \mathrm{~F}_{6}, \mathrm{Cl}_{2}, \mathrm{HBr}, \mathrm{SF}_{6}, \mathrm{O}_{2}, \mathrm{He}$, and $\mathrm{N}_{2}$ purge gases are supplied. The TCP 9400SE etcher has two built-in photodiode endpoint detectors based on Optical Emission Spectroscopy (OES): 405 and $520 \mathrm{~nm}$ wavelength bandpass OES sensors. The $405 \mathrm{~nm}$ wavelength matches the $\mathrm{SiN}$ and $\mathrm{AlCl}$ emission lines, whereas the $520 \mathrm{~nm}$ matches the $\mathrm{C}_{2}, \mathrm{CO}, \mathrm{SiCl}$, and $\mathrm{O}_{2}$ emission lines. To minimize disturbance from the process chamber being exposed to the atmosphere and absorbing water vapor, an entrance and exit loadlock are provided. To acquire data and control the etching process, a PC running LabVIEW was piggybacked onto the Nvision system, which is an original operating system of the Lam TCP 9400SE. A custom LabVIEW-based control program, EMACS, is monitors and controls the etching process, while other processes, such as wafer loading/unloading, pumping, and venting, are operated by Lam's Nvision system.

\section{A.2 Broadband RF sensor hardware and software}

A Hewlett Packard 8753B Vector Network Analyzer, calibrated with a Hewlett Packard 8732B type N calibration kit, was used to collect the frequency response of the plasma etching system. This analyzer can sweep and collect frequency responses ranging from $300 \mathrm{KHz}$ to $3 \mathrm{GHz}$. A tungsten probe was inserted in an aluminum cylinder contained in a quartz tube. About one-inch of the tungsten probe tip from the end of the aluminum cylinder was exposed to the atmosphere inside of the quartz tube (Fig. 5). The tube was placed inside the etcher, however, it did not physically touch the plasma. Contacting the plasma may improve the sensitivity of the RF sensor, however, it may increase the 
possibility of contamination of the chamber. The RF sensor control system based on the LabVIEW program communicates with the network analyzer, and thus the collected RF data can be transferred to the control system. The current Broadband RF sensor setup is able to collect the RF data up to a $3 \mathrm{~Hz}$ sampling rate. This rather slow sampling rate mainly comes from hardware limitations: slow computing speed retarded by the broad range of the frequency sweep, and GPIB communication delay. The sampling frequency can be improved at the cost of the frequency sweeping range.

\section{B. Wafer Structure and Baseline Main Etch Recipe}

A six-inch blank test wafer consisting of $500 \mathrm{~nm}$ n-doped poly-Si on top of $30 \mathrm{~nm}$ oxide on a Si substrate was etched. The native oxide layer on the test wafer was removed by 15 sec of $\mathrm{C}_{2} \mathrm{~F}_{6} \mathrm{Break}$ Through (BT) etch. The fault detection experiment was conducted during the Main Etch (ME) which uses $\mathrm{Cl}_{2}$ and $\mathrm{HBr}$ gases. The typical etch rate for n-doped poly-Si in BT was about $36 \mathrm{~nm} / \mathrm{min}$, and $201 \mathrm{~nm} / \mathrm{min}$ in ME. The ME recipe provided 10:1 etch selectivity of poly-Si over oxide. The details of the BT and ME recipes are shown in Table I. To extract RF sensor signal fingerprints under various sensor fault conditions, we designed the following experiment.

\section{Design of Experiment}

\section{A. Selection of Sensors}

The standard Lam TCP 9400SE is equipped with various sensors to assure stable etch. We selected three types of sensors for the sensor fault detection test: two power measurement sensors, two flow rate sensors, and one pressure sensor. These five sensors were selected because they are particularly prone to fault and their impact on the process result is significant. The two power measurement sensors are found in two power generators: one in the Transformer Coupled Power (TCP) and one in the BIAS power generator. Sensor faults in power measurement typically come from phase or magnitude detector errors in the matching network. The matching network maximizes the power delivered to the etching system from the power generator. For our test, the flow rate sensors for $\mathrm{Cl}_{2}$ and $\mathrm{HBr}$ were selected. The sources of flow rate sensor faults are gas leak, mass flow controller miscalibration, or sensor drift. Finally, the pressure sensor regulates the chamber pressure. Errors in this sensor are the result of either miscalibration or sensor drift. 


\section{B. Experimental Design}

After selecting sensors for the fault detection test, we proceeded with the experiment to collect distinctive RF fingerprints under the respective sensor faults. In this experiment, we assumed first the occurrence of only one sensor fault at a time. And secondly, we assumed that sensor fault can be simulated by a change in machine input setting. Five selected machine inputs, each corresponding to the specific sensor fault, were varied one at a time to simulate a sensor fault. TCP, BIAS power, and $\mathrm{HBr}$ flow rate were varied $\pm 10 \%, \pm 15 \%$, and $\pm 25 \%$ from their respective nominal values of $250 \mathrm{~W}, 180 \mathrm{~W}$, and $75 \mathrm{sccm}$, respectively. Since pressure and $\mathrm{Cl}_{2}$ flow rate settings are small values, 10 mTorr and $15 \mathrm{sccm}$ respectively, their respective nominal values were changed by $\pm 20 \%$, $\pm 30 \%$, and $\pm 40 \%$. Based on the first assumption, only one sensor fault occurs at a time, only one machine input was changed while the other machine inputs were fixed to nominal values. To minimize the number of test wafers used, we etched a single wafer with several different etch recipes without breaking the established vacuum. To establish a new setpoint from the previous one, each etch condition was maintained for $15 \mathrm{sec}$ without ignition of plasma to stabilize the pressure or flow rate to a new setpoint. After the stabilization phase, plasma was ignited for 15 sec. The entire experiment was repeated and averaged to decrease random error. To investigate the stability and repeatability of the Broadband RF sensor response, 30 base line etches were included randomly. Furthermore, each etch setpoint was run in random order to minimize bias error where a numerical value tends to remain constant or follow a consistent pattern over a number of experimental runs.

\section{Broadband RF Sensor Signal Parameterization}

A wafer undergoes a transitioning and settling period during the first couple of seconds after plasma ignition. In order to obtain reliable and repeatable RF sensor fingerprints for each etch condition, the last 5 sec of RF sensor signal of the 15 sec etch period were averaged. The HP network ana-

lyzer swept $0.5 \sim 2.75 \mathrm{GHz}$ and collected 201 data points of log magnitude, $\log (\Gamma)$, and 201 data points of phase, $\arg (\Gamma)$, of the reflection coefficient. The enormous number of RF data points causing an over-determined regression problem in modeling procedure was tackled by means of RLC circuit parameterization [15]. Each RF sensor signal peak was modeled as a single RLC circuit with the components connected in series. The admittance of the RLC circuit is:

$$
Y(s)=\frac{\frac{1}{L} s}{s^{2}+\frac{R}{L} s+\frac{1}{L C}} .
$$


In our parameterization procedure, we used the natural frequency $\left(\omega_{n}\right)$, quality factor $(\mathrm{Q})$, and resistance $(\mathrm{R})$ which are based on expressing eq. (6) as:

$$
Y(s)=\frac{\frac{2 \omega_{n}}{R Q} s}{s^{2}+\frac{2 \omega_{n}}{Q} s+\omega_{n}^{2}} .
$$

Figure 6 illustrates the Broadband RF sensor signal parameterization result. The magnitude and phase of each RF peak were simultaneously fitted to the simulated data to find a set of $\omega_{n}, \mathrm{Q}$, and $\mathrm{R}$ which minimizes R-squared modeling error.

Under the nominal ME condition, three peaks were observed near 1.1, 1.4, and $2.0 \mathrm{GHz}$ respectively. However, the third peak near $2 \mathrm{GHz}$ was noisy, and not as responsive as the low $(1 \mathrm{GHz})$ or medium range $(1.4 \mathrm{GHz})$ peak. Therefore, we excluded it from the valid $\mathrm{RF}$ sensor signal. Since $\mathrm{R}$ is too sensitive to plasma conditions and fluctuates under stable etching conditions, we excluded the R from the valid RF sensor signal as well. In this study, therefore, we chose two peak frequencies, $\omega_{n 1}$ and $\omega_{n 2}$, and two $\mathrm{Q}$ values, $\mathrm{Q}_{1}$ and $\mathrm{Q}_{2}$, of low and medium range peaks as valid $\mathrm{RF}$ information. The following section provides an analysis of the Broadband RF sensor signals under simulated sensor faults.

\section{Experimental Result}

\section{A. RF Sensor Signal vs. Sensor Fault}

In a dual-power-source plasma etching system, Transformer Coupled Power (TCP) controls plasma density. The plasma density can be increased by supplying more TCP to the etching system. The RF peak frequency $\left(\omega_{n}\right.$, which has the lowest reflection coefficient) is an important indicator of the plasma density variation. Thus, we can detect the delivered TCP change to the etching system by observing the RF peak frequency variation. The plasma density can be approximated with the RF peak frequency of the reflection coefficient [16]:

$$
n_{e}=\frac{\omega^{2} \epsilon_{o} m_{e}}{q^{2}} \approx 1.24 \mathrm{E}-8 \cdot f^{2},
$$

where $f=\omega / 2 \pi$, the frequency having the lowest reflection coefficient, $e_{o}$ is free space permittivity, 8.85E-12 F/m, $m_{e}$ is the mass of electron, $9.109 \mathrm{E}-31 \mathrm{Kg}$, and $q$ is the electron charge magnitude, 1.602E-19 C. Based on eq. (8), the calculated plasma density of the Lam TCP 9400SE was 1.0E10 2.1E10 under $188 \sim 312 \mathrm{~W}$ of TCP, $180 \mathrm{~W}$ of BIAS power, $10 \mathrm{mTorr}$ of pressure, $15 \mathrm{sccm}$ of $\mathrm{Cl}_{2}$, and $75 \mathrm{sccm}$ of $\mathrm{HBr}$ flow rate. The calculated plasma density was lower than the actual plasma density measured with a Langmuir probe, but was the same order of magnitude. Furthermore, the linear increase of estimated plasma density with TCP is consistent with the findings in the literature [17-19]. 
Figure 7 illustrates a variation of peak frequency with various TCP settings. With TCP, the peak frequency was increased, and the gap between the two peak frequencies of each Broadband RF sensor signal was also slightly increased. With $\pm 25 \%$ change in TCP, the peak frequency was changed about $\pm 16 \%$ (Fig. $8(\mathrm{a})$ ). Although BIAS power strongly affects ion energy, it does not disturb plasma density significantly $[18,19]$. In our experiment, observation confirmed that the peak frequency change with $\pm 25 \%$ BIAS power variation was only $\pm 1 \%$, showing a small correlation between the two (Fig. $8(\mathrm{~b}))$.

Pressure is another actuator controlling plasma density. However, in the pressure range in this experiment, 6 mTorr $\sim 14$ mTorr, only a slight increase of peak frequency was observed: less than \pm $5 \%$ under $\pm 40 \%$ pressure variation (Fig. 8 (c)). In fact, the peak frequency was maximized when pressure reached 12.5 mTorr, then started to decrease. The peak frequency variation with $\mathrm{Cl}_{2} \mathrm{Or} \mathrm{HBr}$ flow rate was insignificant (Fig. $8(\mathrm{~d})$ and (e)). In both these cases, less than $\pm 2 \%$ variation of the peak frequency was observed under $\pm 40 \%$ or $\pm 25 \%$ of $\mathrm{Cl}_{2}$ or $\mathrm{HBr}$ flow rate variation, respectively. In short, the peak frequency in the Broadband RF sensor signal is very sensitive to the plasma density variation. Therefore, changes in etch condition, which significantly affect the plasma density variation (e.g., TCP), can be easily detected by observing the peak frequency change of the Broadband RF sensor.

The other observed RF sensor signal is $\mathrm{Q}$. The variations of $\mathrm{Q}$ with various machine input settings are well illustrated in Fig. 8. In most instances, $Q_{1}$ and $Q_{2}$ variations with machine input have a seemingly random pattern and are not yet clearly understood. However, $\mathrm{Q}_{1}$ with pressure and $\mathrm{Q}_{2}$ with $\mathrm{HBr}$ are sensitive to the variation of its machine input and they increases linearly. Except the low flow rate, $\mathrm{Q}_{2}$ is also very sensitive to the $\mathrm{Cl}_{2}$ flow rate. However, because the $\mathrm{Q}_{1}$ or $\mathrm{Q}_{2}$ variations under each machine input variation overlap each other, it is difficult to trace the exact cause of the machine input variation by observing $\mathrm{Q}_{1}$ or $\mathrm{Q}_{2}$.

When we select a Broadband RF sensor signal for a specific sensor fault detection, this sensor signal should be very sensitive to that sensor fault but insensitive (orthogonal) to the other sensor faults. As explained previously, $\omega_{n 1}$ and $\omega_{n 2}$ are excellent TCP fault indicators. However, none of the four RF sensor signals are directly useful to detect the other types of sensor faults. When a specific sensor fault occurs, the actual machine input value corresponding to that sensor in the etching system is different from the original setting due to the malfunctioning sensor. Thus, if we accurately estimate the actual machine input value in real time, we can effectively identify which sensor is at fault by measuring 
the amount of drift from the nominal value. In the following section, we describe how we achieved sensor fault detection by using RF sensor measurement to estimate actual machine input values. First, the general procedure of machine input estimation is presented and followed by a description of TCP measurement sensor fault detection by TCP estimation. Then, the pressure sensor fault detection, which cannot be detected with raw RF sensor signal observation, is investigated. Lastly, a validation experiment used for TCP and pressure sensor fault detection is reviewed.

\section{B. Sensor Fault Detection via Machine Input Estimation}

Sensor fault constitutes a drift from the original machine input setting. Thus, by estimating the amount of drift from the nominal value, we can detect the sensor at fault. For the success of this sensor fault detection method, an accurate estimation of the actual machine input value is critical. First, to obtain more dynamics of RF sensor signal under various machine input variations within the constraints of time and budget, we interpolated 7 setpoints of each machine input in the experiment to obtain 100 setpoints. Since setpoints and signals are expressed in different units, they were normalized to the respective nominal values. Then, the standard deviation of each $\mathrm{RF}$ sensor signal was calculated from the 30 baseline etches. The standard deviation was multiplied by randomly generated Gaussian noise with mean zero and standard deviation one. This was assumed to be measurement noise. Finally, we added the measurement noise to the interpolated RF sensor signal. We repeated this procedure, adding measurement noise to the interpolated RF sensor signal, until $3000 \mathrm{RF}$ sensor signal data sets were generated. Figure 9 illustrates the maximum and minimum values of the generated RF sensor signal under the five machine input variations simulating sensor faults.

For the machine input estimation, a full quadratic model employing a subset selection methodology, forward selection and backward elimination, was built. To improve modeling accuracy, a $520 \mathrm{~nm}$ Optical Emission Spectroscopic (OES) sensor signal was added to the four RF sensor signals. In the modeling procedure, we first selected 10 modeling variables out of 20 in an order that achieved the highest R-squared value in the forward selection step. In short, one additional variable was selected among the possible modeling variables when the R-squared value was maximized when it was selected. Once the desired number of the modeling variables had been selected, in the backward elimination step the selected modeling variables were removed one by one in the order of least damaging to the model accuracy. When the probability of decrease in model size by one was larger than 0.05 under an F-test, we stopped the elimination step and used the modeling variables that were not eliminated for 
the model. The final model typically consisted of $4 \sim 7$ variables.

\section{B.1 TCP Measurement Sensor Fault Detection}

Following the modeling procedure as described in the previous section, first the TCP estimation model was built. With $\mathrm{R}^{2}=0.94$ and $\pm 5 \%$ modeling error, the final TCP estimation model has a constant, $\omega_{n 1}, \omega_{n 2}$, and $Q_{1}$ :

$$
\widehat{\mathrm{TCP}}=0.1985+0.8574 \omega_{\mathrm{n} 2}-0.6529 \mathrm{Q}_{1}+0.9510 \omega_{\mathrm{n} 1} \mathrm{Q}_{1}
$$

where $\omega_{n 1}$ and $\omega_{n 2}$ are low and medium range RF peak frequencies respectively, and $Q_{1}$ is a quality factor of the low range RF peak. All of the modeling variables $\left(\omega_{n 1}, \omega_{n 2}\right.$, and $\left.Q_{1}\right)$ and estimated TCP $(\widehat{T C P})$ are normalized values. Figure 10 illustrates the resulting minimum and maximum values of the estimated TCP by substituting the 3000 RF data sets for the eq. (9). Ideally, the estimated TCP should be a straight line in the region labeled as "TCP" since TCP increased linearly. It should be flat in the regions of BIAS power, pressure, and gas flow rate because TCP was set to the nominal value while those machine inputs were changed.

We then set two hypotheses for TCP fault detector, $H_{0}$ : no TCP fault and $H_{1}$ : TCP fault. The input to the fault detector was determined to be $|1-\widehat{T C P}|$ since we detect the sensor fault by measuring the amount of drift of actual machine input from the nominal setpoint. To check the conditional probability density function of the input to the fault detector, histograms of "no TCP Fault" and "TCP Fault" were evaluated (Fig. 11).

These histograms illustrate the distribution of $|1-\widehat{T C P}|$ under "no TCP Fault" and "TCP Fault" hypotheses, respectively. Although the distribution of "no TCP Fault" is a single-sided Gaussian form, the distribution of "TCP Fault" is difficult to define. Therefore, we employed a non-parametric detection method instead of a parametric detection method which requires an exact distribution function for each detector input. Based on a sign test, which is a simple non-parametric detection method, the following decision criteria were set to determine the TCP measurement sensor fault:

$$
\begin{array}{ll}
H_{0} \text { : no TCP Fault, } & \frac{\left|T C P_{\text {nom }}-\widehat{T C P}\right|}{T C P_{\text {nom }}}<\gamma \\
H_{1} \text { : TCP Fault, } & \frac{\left|T C P_{\text {nom }}-\widehat{T C P}\right|}{T C P_{\text {nom }}} \geq \gamma,
\end{array}
$$

where $\widehat{T C P}$ is a normalized estimated TCP, $T C P_{\text {nom }}$ is a normalized nominal TCP setting which is 1 , and $\gamma$ is a threshold with a value larger than 0.05 . The TCP estimation model had approximately \pm 5 $\%$ modeling error. This means that the value of TCP estimation within $\pm 5 \%$ from the nominal TCP 
does not necessary imply TCP fault. Thus, we designated the estimated TCP within $\pm 5 \%$ variation from the nominal TCP as the "nominal" etch condition. For this reason, the minimum threshold $(\gamma)$ that determines "no TCP Fault" was set to 0.05.

We assumed that $P$ (individual sensor fault occurred) is $10^{-4}$, and that each sensor fault occurrence is mutually exclusive and equally likely for the further calculation of $P_{D}$ and $P_{F}$. The fault detection probability $\left(P_{D}\right)$ is defined as:

$$
P_{D}=P(\text { TCP fault detected | TCP fault occurred }),
$$

and, the false alarm rate $\left(P_{F}\right)$ is:

$$
P_{F}=P\left(\text { TCP fault detected | TCP fault occurred }{ }^{\mathrm{c}}\right),
$$

where superscript "c" means the set complementary. And TCP fault occurred ${ }^{c}$ is defined as:

TCP fault occurred ${ }^{\mathrm{c}} \triangleq$ Nominal $\cup$ Bias power sensorfault $\cup$ Pressure sensor fault $\cup$ $\mathrm{Cl}_{2}$ flow rate sensor fault $\cup \mathrm{HBr}$ flow rate sensor fault .

By Bayes rule, $P_{F}$ can be rewritten as:

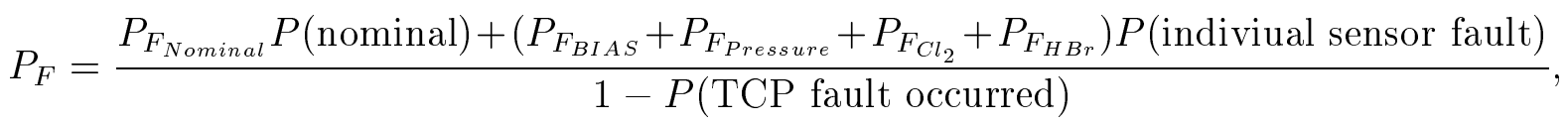

where

$$
\begin{gathered}
P_{F_{\text {Nominal }}}=P(\text { TCP fault detected } \mid \text { Nominal }) \\
P_{F_{B I A S}}=P(\text { TCP fault detected } \mid \text { BIAS power fault occurred }) .
\end{gathered}
$$

$P_{F_{\text {Pressure }}}, P_{F_{C l_{2}}}$, and $P_{F_{H B r}}$ can be expressed in the same manner. Since we assume that each sensor has the same probability of fault occurrence, $P($ individual sensor fault $)=1 \mathrm{E}-4$, and thus, $P($ nominal $)=1$ $5 \cdot P($ individual sensor fault $)=0.9995$.

Figure 12 illustrates the performance of the TCP fault detector in a graph of detection probability $\left(P_{D}\right)$ vs. probability of false alarm $\left(P_{F}\right)$. First, $P_{D}$ was calculated when $\pm 6 \%, \pm 7 \%, \pm 8 \%$, and \pm $9 \%$ TCP error from the nominal value occurred with threshold $(\gamma)$ ranging from 0.05 to 0.35 . Then, $P_{F}$ was calculated as a function of the threshold $(\gamma=0.05 \approx 0.35)$. When a TCP measurement sensor fault caused the drift of the actual TCP to $\pm 8 \%$ deviation from its nominal setting, this TCP fault could be detected with 0.99 detection probability when the false alarm rate was set to $1 \mathrm{E}-4$. The TCP fault detector provided excellent fault detection accuracy when TCP deviation by a TCP measurement sensor fault was more than $\pm 8 \%$ from its nominal value. 


\section{B.2 Pressure Sensor Fault Detection}

Pressure sensor fault detection, which cannot be addressed with raw RF sensor signal observation, was also investigated. In the same manner as we designed for TCP fault detection, we built a pressure estimation model based on forward selection and backward elimination, employing Broadband RF sensor signals and a $520 \mathrm{~nm}$ OES signal. The final pressure estimation model is:

$$
\text { pressure }=0.4389+1.5931 w_{n 1}-1.6611 O E S+0.1451 w_{n 1} Q_{1}+0.4862 w_{n 2} \text { OES }
$$

with $R^{2}=0.845$. The estimated pressure (p⿹勹巳esure $)$ and all of the modeling parameters are normalized values. Figure 13 illustrates the maximum and minimum values of the estimated pressure by evaluating eq. (11) with each of the 3000 data sets of RF sensor signals. We allowed $\pm 10 \%$ of fault margin in the pressure sensor. The following decision criteria were set to determine a pressure sensor fault:

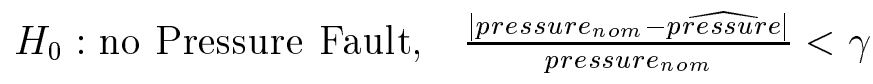

$$
\begin{aligned}
& H_{1} \text { : Pressure Fault, } \quad \frac{\mid \text { pressure }_{\text {nom }}-\text { pressure } \mid}{\text { pressure }_{\text {nom }}} \geq \gamma,
\end{aligned}
$$

where pressure is the estimated pressure, pressure $_{\text {nom }}$ is a nominal TCP setting, and $\gamma$ is a threshold to be determined $(\gamma>0.1)$. Assumptions that $P$ (individual sensor fault occurred) is $10^{-4}$, and that each sensor fault occurrence is mutually exclusive and equally likely were also applied. Fig. 14 illustrates the histograms of $\mid$ pressure $_{\text {nom }}$ - p $\widehat{\text { ressur }}|=| 1-$ pressure $\mid$ under "no Pressure Fault" and "Pressure Fault". Figure 15 illustrates the performance of the pressure sensor fault detector in a graph of detection probability $\left(P_{D}\right)$ vs. probability of false alarm $\left(P_{F}\right)$. When $\pm 17.5 \%$ of pressure error occurred, the pressure sensor fault could be detected with 0.92 detection probability with a $1 \mathrm{E}-4$ false alarm rate. More than $\pm 20 \%$ drift from the nominal value causing pressure sensor fault could be detected very accurately: $P_{D} \approx 1$ and $P_{F} \approx 0$. It is worth noting that the $P_{D}$ and $P_{F}$ were calculated based on our DOE, which puts bounds on the size of the errors in the remaining variables.

\section{B.3 Validation Experiment}

To check the robustness of the TCP and pressure sensor fault detector, a validation experiment was performed 5 months after the original fault detection experiment on the same etching system. The etch recipe shown in Table II was used for this validation experiment. One machine input was changed at a time. The etch conditions of nine nominal, eighteen TCP error (eight $225 \mathrm{~W}$ and ten $275 \mathrm{~W}$ ), nineteen pressure error (ten 8 mTorr and nine 12 mTorr), four BIAS power (125 W), three HBr flow 
rate error $(86 \mathrm{sccm})$, and two $\mathrm{Cl}_{2}$ flow rate error $(7 \mathrm{sccm})$ were included. Figure 16 illustrates the performance of the TCP and pressure sensor fault detectors in the validation experiment. The dashed lines in the figure are the upper and lower bounds of the nominal TCP and pressure fault margins corresponding to $\pm 5 \%$ and $\pm 10 \%$, respectively. In the TCP measurement sensor fault detection, the detector identified all TCP faults without generating any false alarm. Whereas, the pressure sensor fault detector generated two false alarms. This was expected because the pressure estimation modeling error was significant in the range of low $\mathrm{Cl}_{2}$ flow rate as shown in Fig. 13. Except for the two false alarms, the pressure sensor fault detector provided excellent fault detection accuracy $\left(P_{D} \approx 1\right)$. The Broadband RF sensor has been proven to be accurate and reliable in the real time detection of sensor faults.

\section{CONClusion}

To reduce a cost in semiconductor manufacturing, it is vitally important to identify and classify the source of machine/sensor error. Thus, the machine down time can be minimized. As an effort to detect sensor errors caused by drift or miscalibration in plasma etching system, a novel sensor fault detection technique employing a Broadband RF sensor was developed. The work presented here focuses on detecting errors in the Transformer Couple Plasma (TCP) measurement and pressure sensor, though the method has potential for several other sensors. We utilized the Broadband RF sensor's unique characteristic to detect the process abnormality caused by sensor fault in real time, and furthermore, could pinpoint the source of the abnormality. To improve the detection sensitivity, we used real-time machine input estimation and compared it from actual recipe setting instead of employing raw RF signal directly. With the use of Broadband RF signal, TCP and pressure could be estimated within \pm $3 \%$ and $\pm 10 \%$ estimation error, respectively. In a main etch process on a Lam TCP 9400SE, \pm 10

$\%$ relative deviations in TCP simulating TCP, and $\pm 25 \%$ in pressure simulating respective TCP and pressure sensor faults in etch can be detected with very high accuracy: Detection probability $P_{D} \approx 1$ and false alarm rate $P_{F} \approx 0$.

\section{ACKnowledgments}

The authors sincerely thank Dr. Garvin (Univ. of Michigan) for help with the Broadband RF measurement. This work was supported in part by the NIST Advanced Technology Program (ATP) under contract No. 70NANB8H4067. 


\section{ReFERENCES}

[1] E. H. Bogardus, V. Bakshi, and J. Gragg, "Sematech projects in advanced process control," IEEE Int. Symp. on Semiconductor Manufacturing, pp. B25-B29, 1997.

[2] S. Leang and C. J. Spanos, "A general equipment diagnostic system and its application on photolithographic sequences," IEEE Trans. Semiconduct. Manufact., vol. 10, no. 3, pp. 329-343, Aug. 1997.

[3] S. Saxena and A. Unruh, "Diagnosis of semiconductor manufacturing equipement and process," IEEE Trans. Semiconduct. Manufact., vol. 7, no. 2, pp. 220-231, May 1994.

[4] N. Chang and C. Spanos, "Continuous equipment diagnosis using evidence integration: An lpcvd application," IEEE Trans. Semiconduct. Manufact., vol. 4, no. 1, pp. 43-51, Feb. 1991.

[5] S. B. Dolins, A. Srivastava, and B. E. Flinchbaugh, "Monitoring and diagnosis of plasma etch processes," IEEE Trans. Semiconduct. Manufact., vol. 1, no. 1, pp. 23-27, Feb. 1988.

[6] A. M. Ison, W. Li, and C. J. Spanos, "Fault diagnosis of plasma etch equipment," IEEE Int. Symposium on Semiconductor on Manufacturing Conference, pp. B49-B52, 1997.

[7] G. S. May and C. J. Spanos, "Automated malfunction diagnosis of semiconductor fabrication equipment: A plasma etch application," IEEE Trans. Semiconduct. Manufact., vol. 6, no. 1, pp. 28-40, Feb. 1993.

[8] M. D. Baker, C. D. Himmel, and G. S. May, "Time series modeling of reactive ion etching using neural networks," IEEE Trans. Semiconduct. Manufact., vol. 8, no. 1, pp. 62-71, Feb. 1995.

[9] B. Kim and G. S. May, "Real-time diagnosis of semiconductor manufacturing equipment using a hybrid neural network expert system," IEEE Trans. Component, Packaging, and Manuf. Technol., vol. 20, no. 1, pp. 39-47, Jan 1997.

[10] J. D. Gibson and J. L. Melsa, Introduction to Nonparametric Detection with Application, IEEE press, Piscataway, NJ, 1996.

[11] J. T. C. Lee H. L. Maynard, E. A. Rietman, D. E. Ibbotson, and E. Dole, "Plasma etching endpointing by monitoring radio-frequency power systems with an artificial neural network," J. Electrochem., vol. 143, no. 6, pp. 2029-2035, June 1996.

[12] J. Scanlan, "Rf and plasma diagnostics for process control and monitoring," AEC/APC Symposium, vol. 1, pp. 237-249, 1999.

[13] C. Garvin, D. S. Grimard, and J. W. Grizzle, "Rf sensing and calibration for real time control of plasma-based deposition and etching," 1998 International Conference on Characterization and Metrology for ULSI Technology, 1998.

[14] M. Klick, W. Rehak, and M Kammeyer, "Plasma diagnostics in rf discharges using nonlinear and resonance effects," Jpn. J. Appl. Phys. Part 1, vol. 36, no. 7B, pp. 4625-4631, July 1997.

[15] C. Garvin and J. W. Grizzle, "An empirical estimate of polysilicon etch rate in a lam 9400 rie tool using broadband rf sensing," J. Vac. Sci. Technol., In press.

[16] S. G. Bilén, J. M. Hass, F. S. Gulczinski III, A. D. Gallimore, and J. N. Letoutchaia, "Resonance-probe measurements of plasma densities in electric-propulsion plumes," 35th AIAA/ASME/SAE/ASEE Joint Propulsion Conference and Exhibit, 1999.

[17] J. H. Keller, "Inductive plasmas for plasma processing," Plasma Sources Sci. Technol., vol. 5, no. 2, pp. 166-172, May 1996.

[18] Y. Ra, S. G. Bradley, and C. Chen, "Etching of aluminum alloys in the transformer-coupled plasma etcher," J. Vac. Sci. Technol., vol. A. 12, no. 4, pp. 1328-1333, Jul/Aug 1994.

[19] R. Patrick, P. Schoenborn, H. Toda, and F. Bose, "Application of a high density inductively coupled plasma reactor to polysilicon etching," J. Vac. Sci. Technol., vol. A. 11, no. 4, pp. 1296-1300, 1993. 


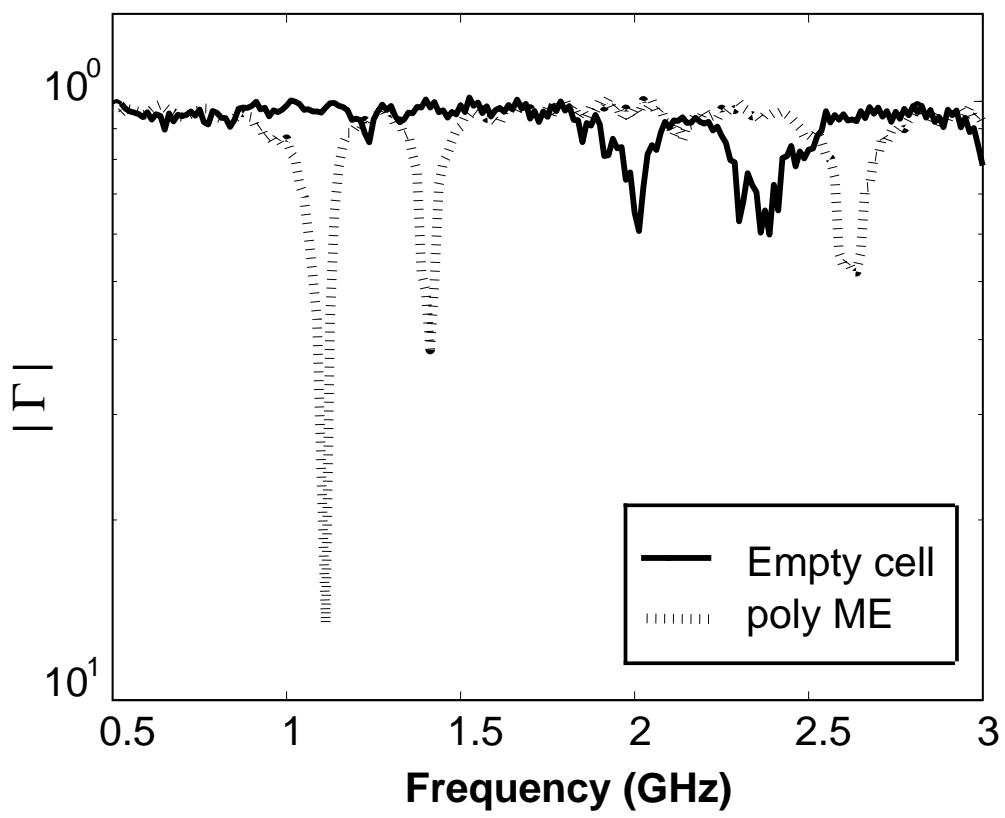

Fig. 1. RF response of an empty chamber and chamber under poly-Si ME. 


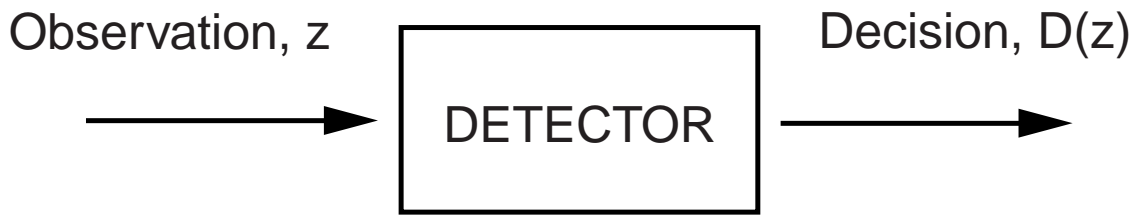

Fig. 2. Generalized detector. 


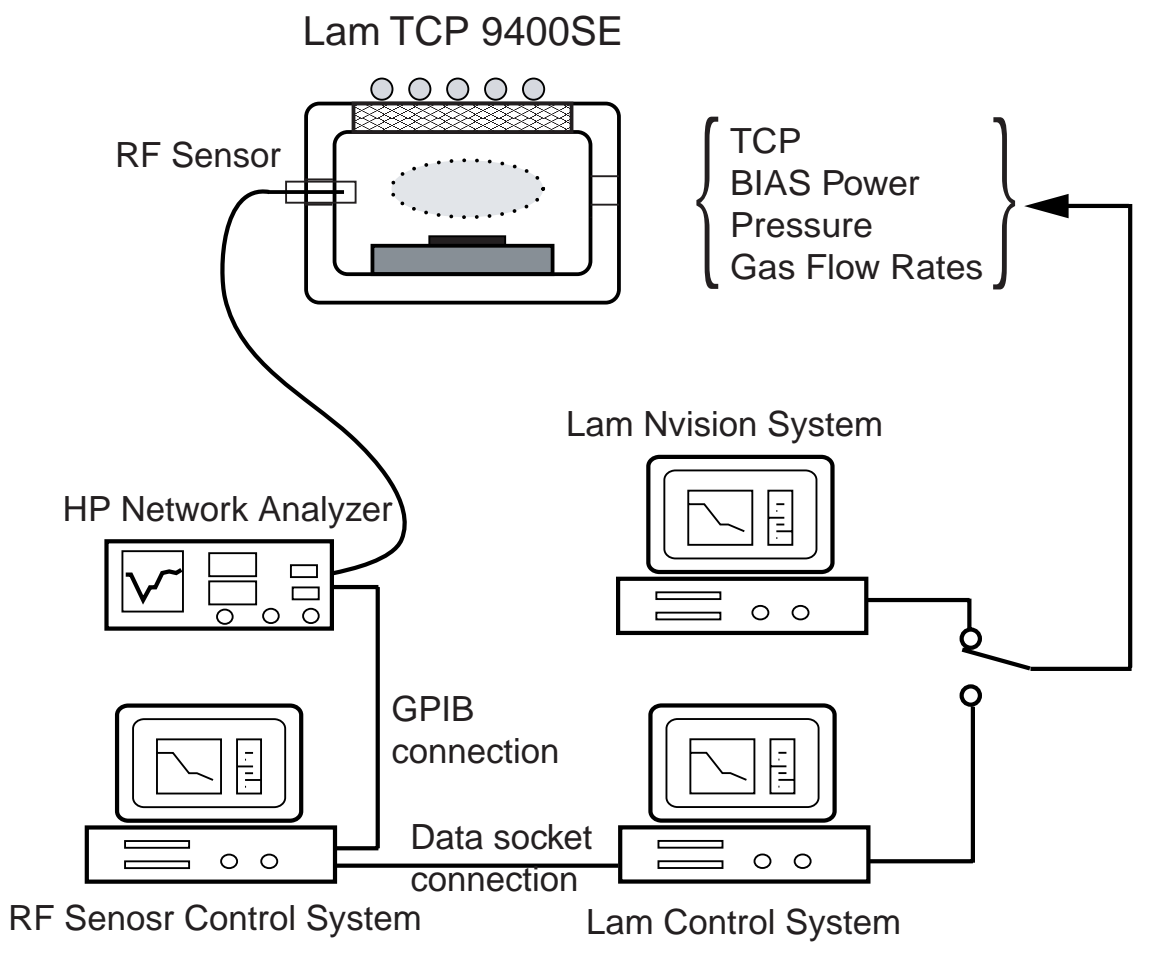

Fig. 3. Schematic view of the Lam TCP 9400SE plasma etching system with a home-built Broadband RF sensor. 


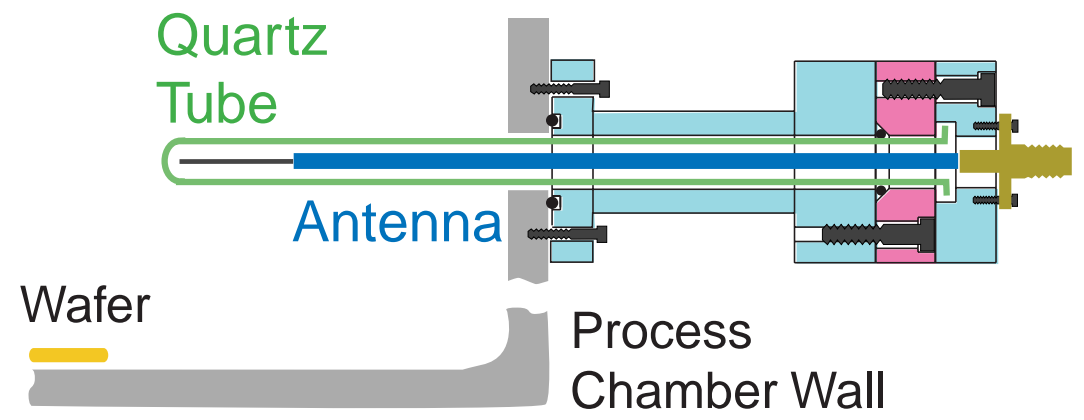

Fig. 4. Schematic of a Broadband RF sensor. 


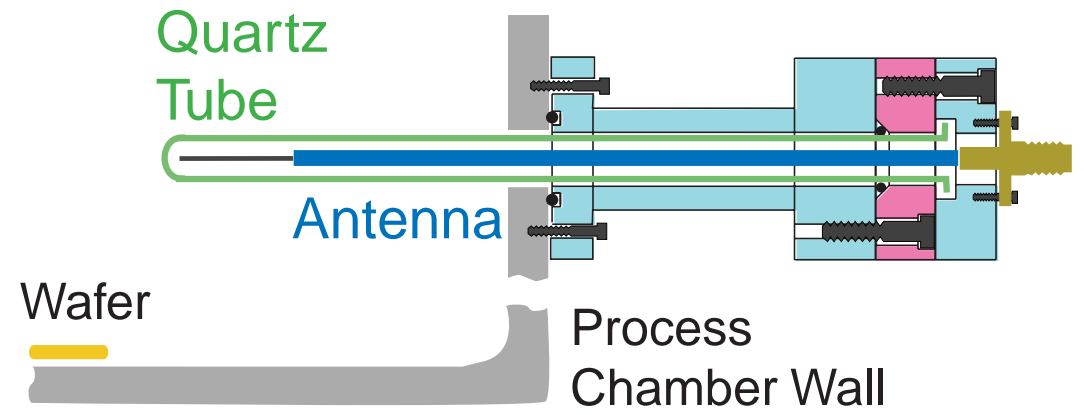

Fig. 5. Schematic of a Broadband RF sensor. 

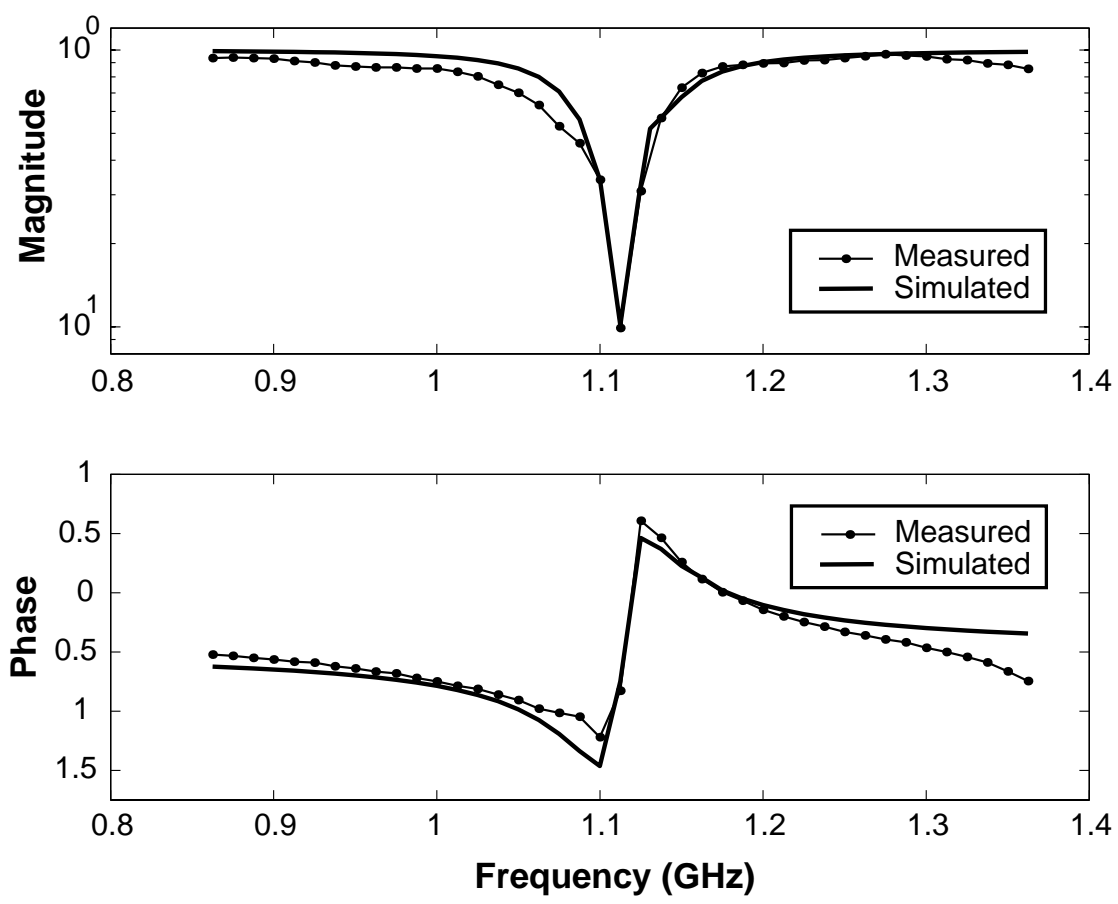

Fig. 6. Broadband RF signal parameterization result. 


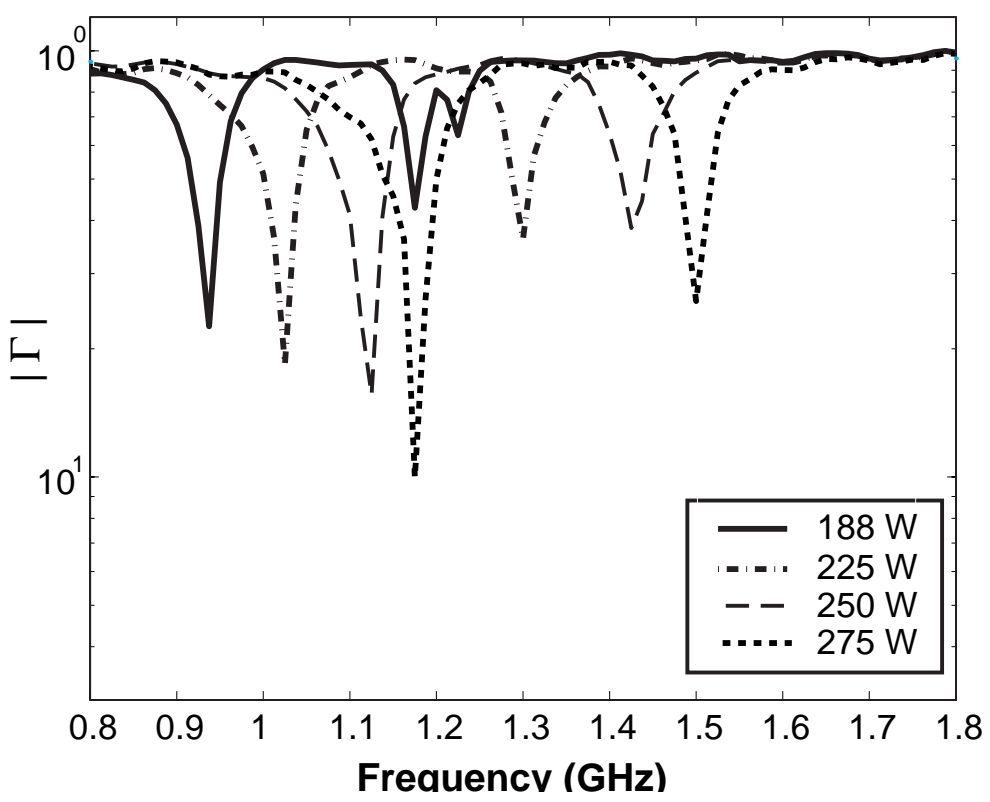

Fig. 7. Variation of an RF peak with various TCP values. 


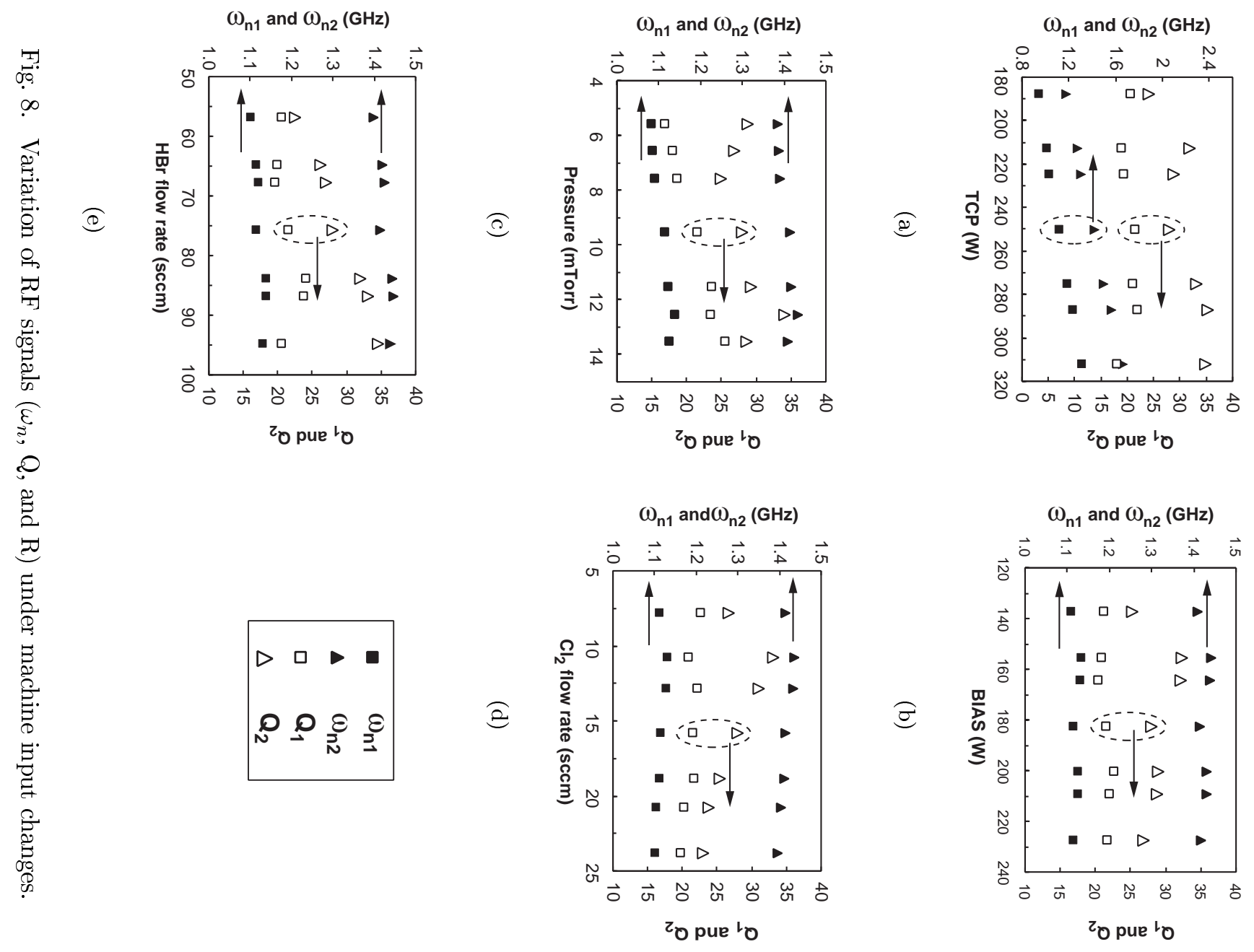




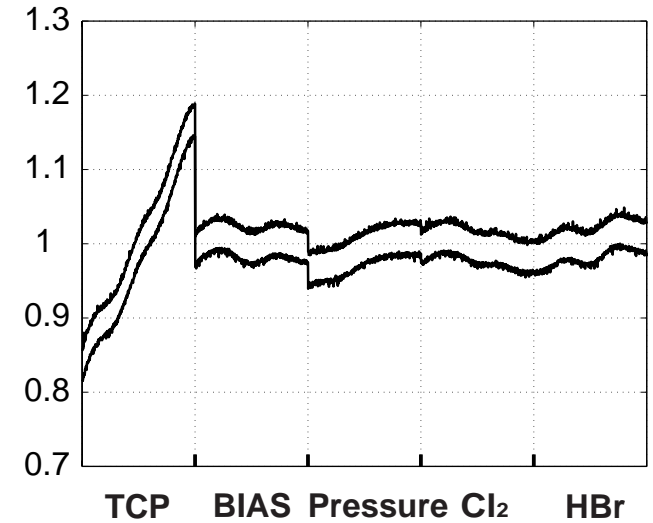

(a) $\omega_{n 1}$

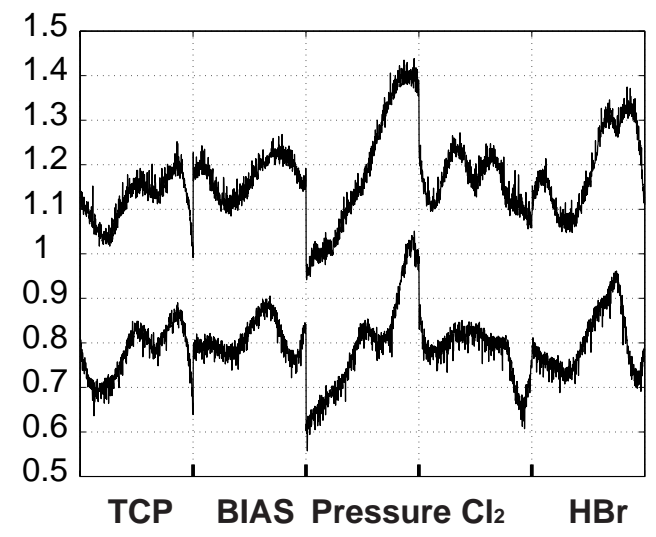

(c) $Q_{1}$

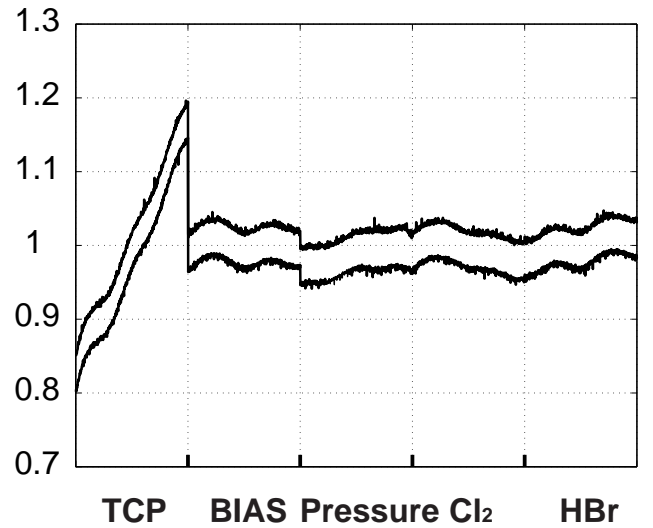

(b) $\omega_{n 2}$

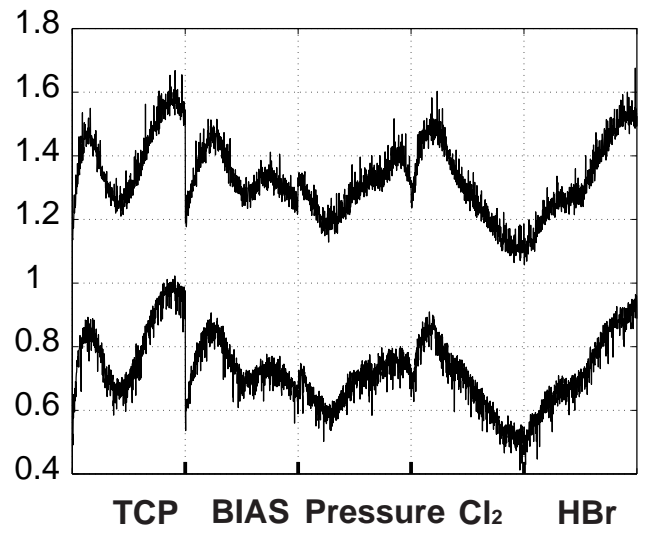

(d) $Q_{2}$

Fig. 9. Maximum and minimum values of RF signals under machine input variation simulating sensor faults. 


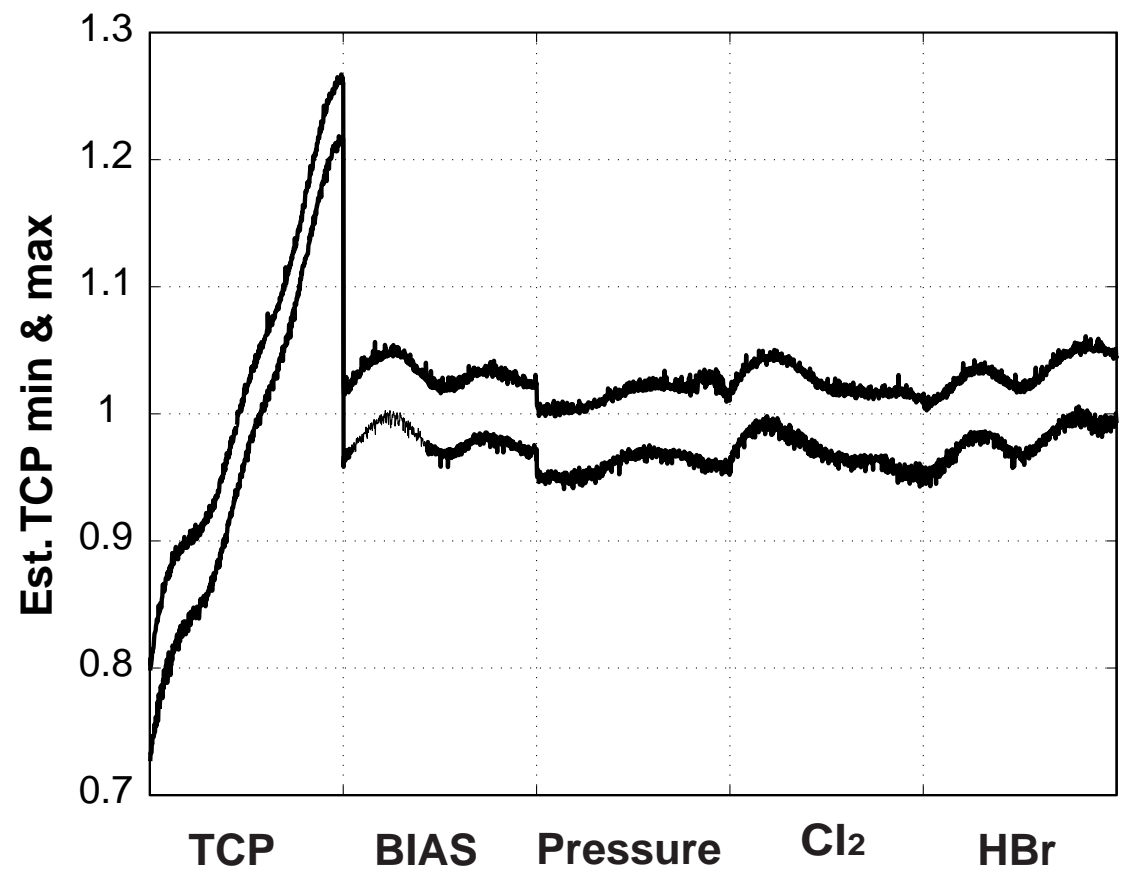

Fig. 10. Normalized Minimum and maximum values of the estimated TCP. 


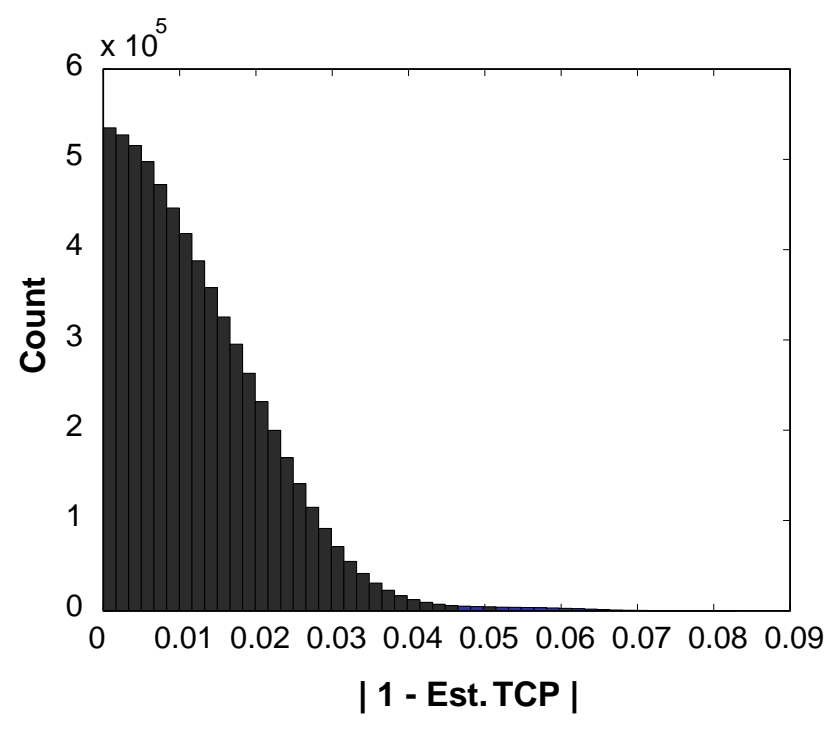

(a) Histogram of No TCP error hypothesis

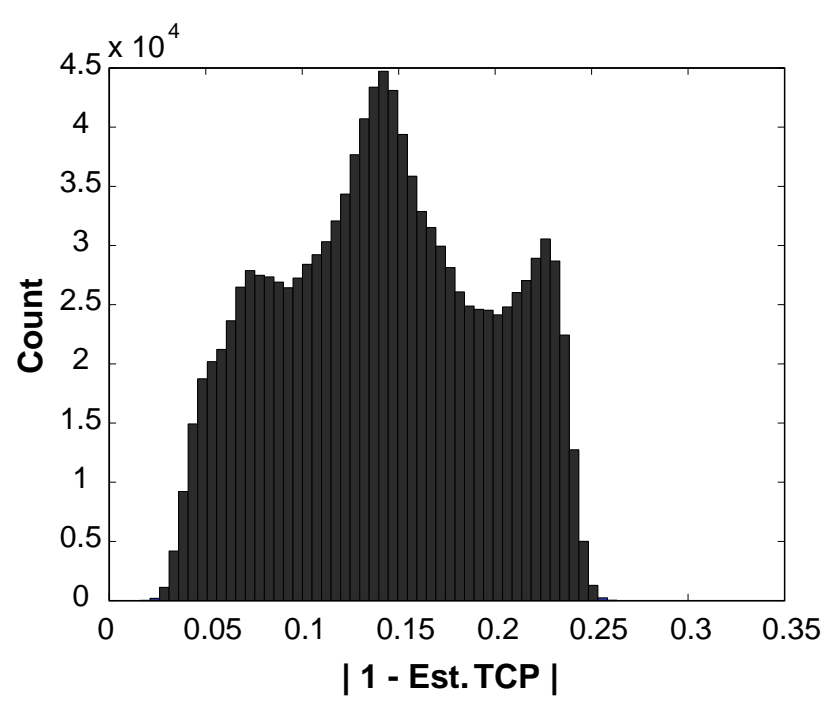

(b) Histogram of TCP error hypothesis

Fig. 11. Histogram of "no TCP Fault" and "TCP Fault" condition. 


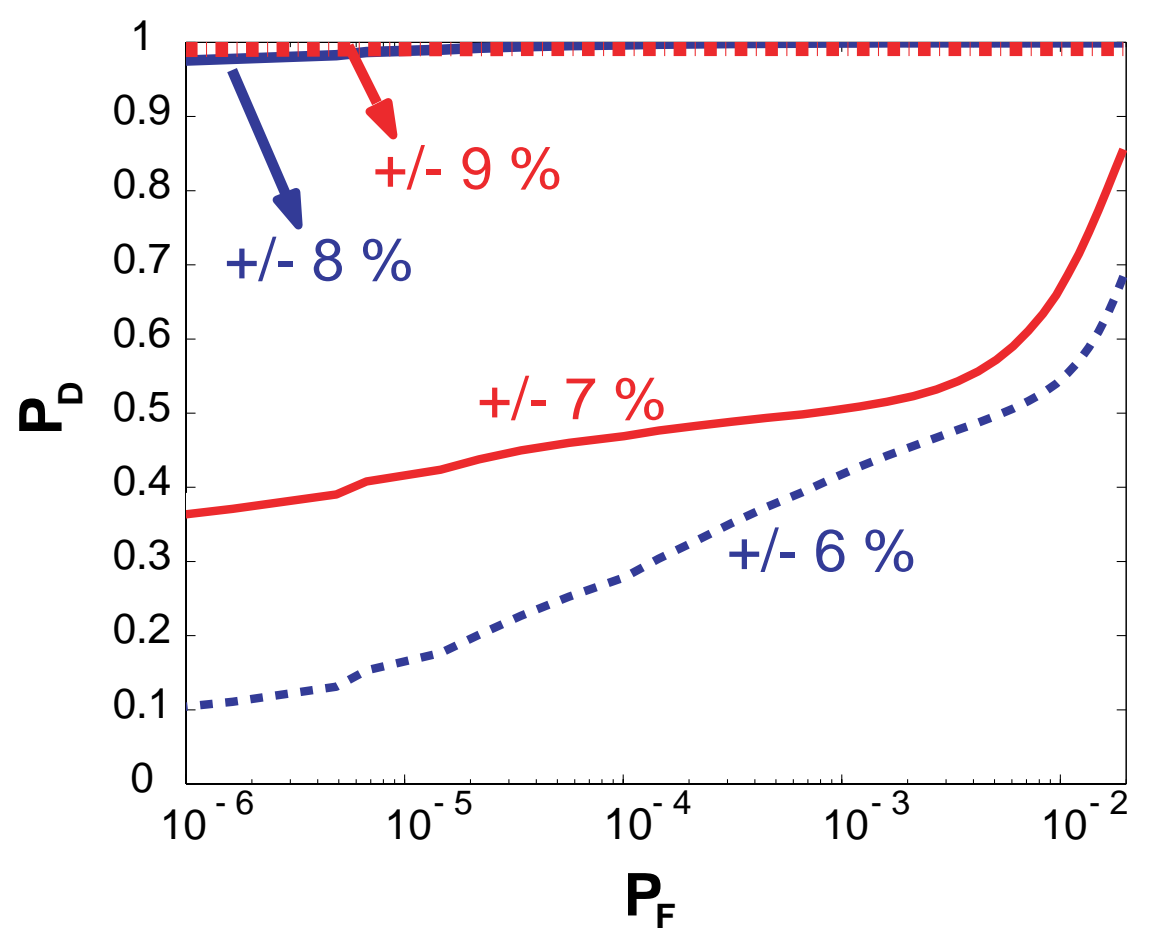

Fig. 12. Receiver Operating Characteristics of a TCP fault detector. 


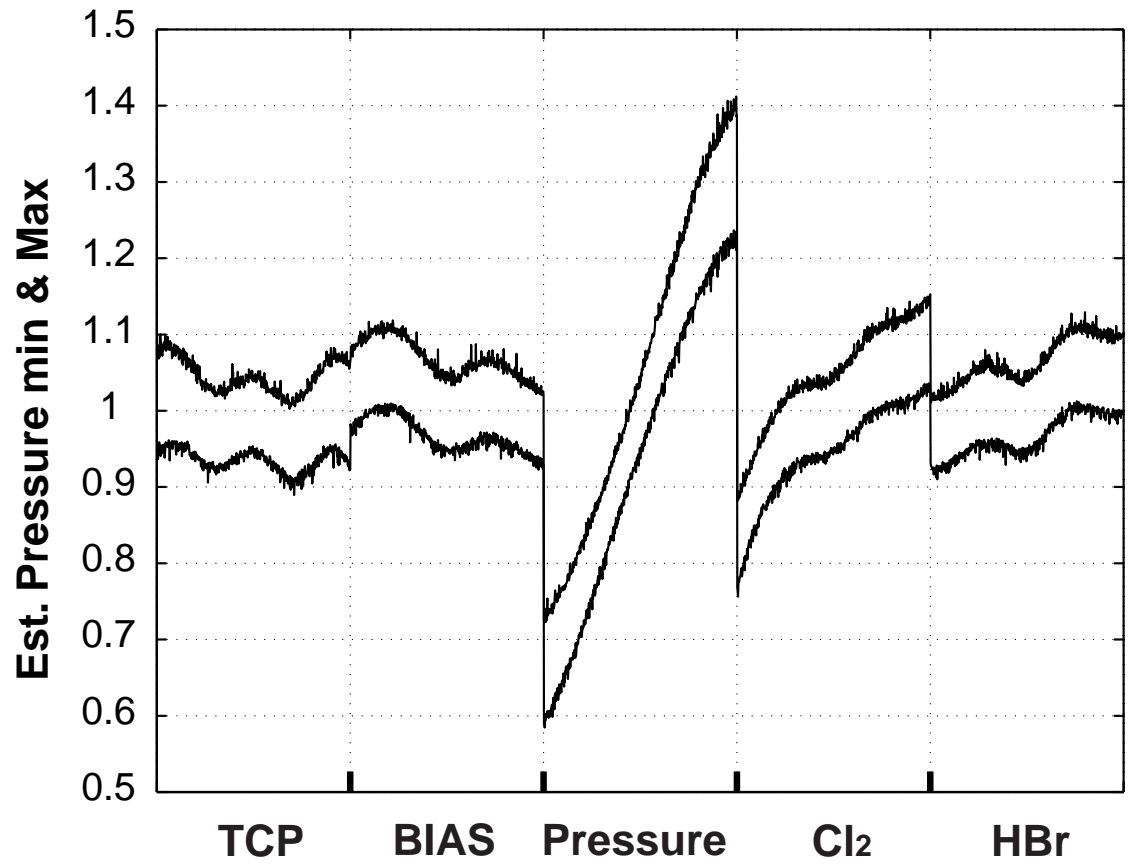

Fig. 13. Normalized minimum and maximum values of the estimated pressure. 


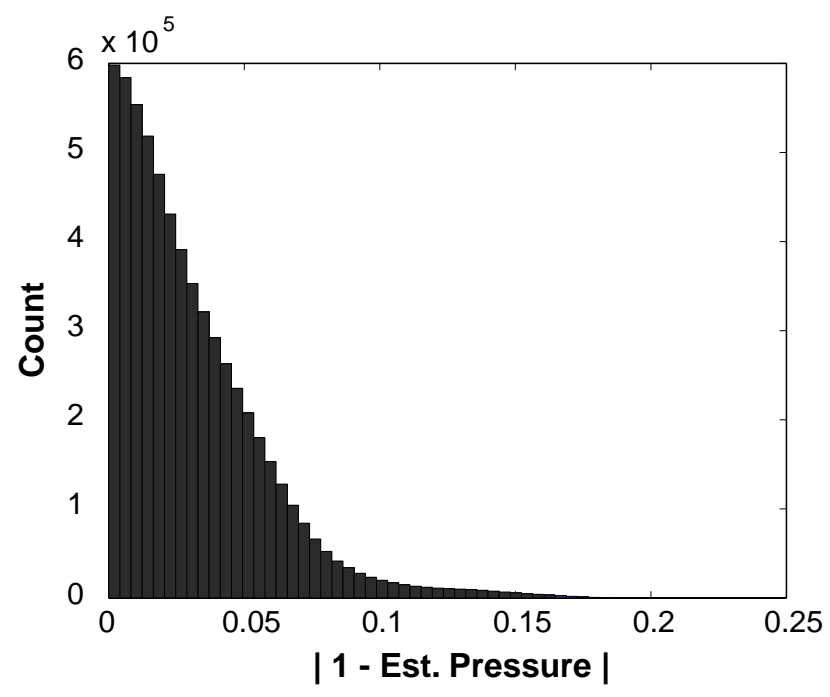

(a) Histogram of No pressure error hypothesis

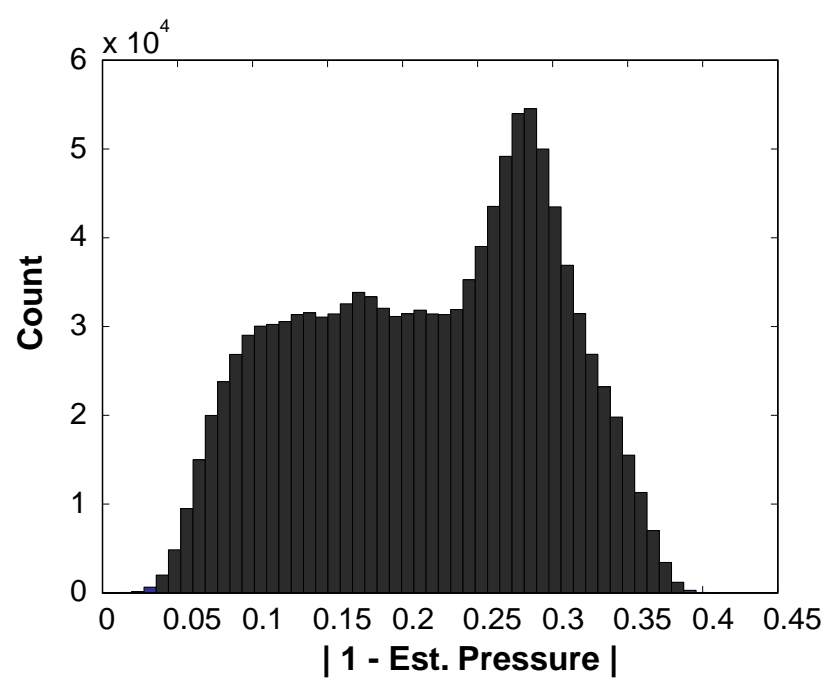

(b) Histogram of pressure error hypothesis

Fig. 14. Histogram of "no Pressure Fault" and "Pressure Fault" hypothesis. 


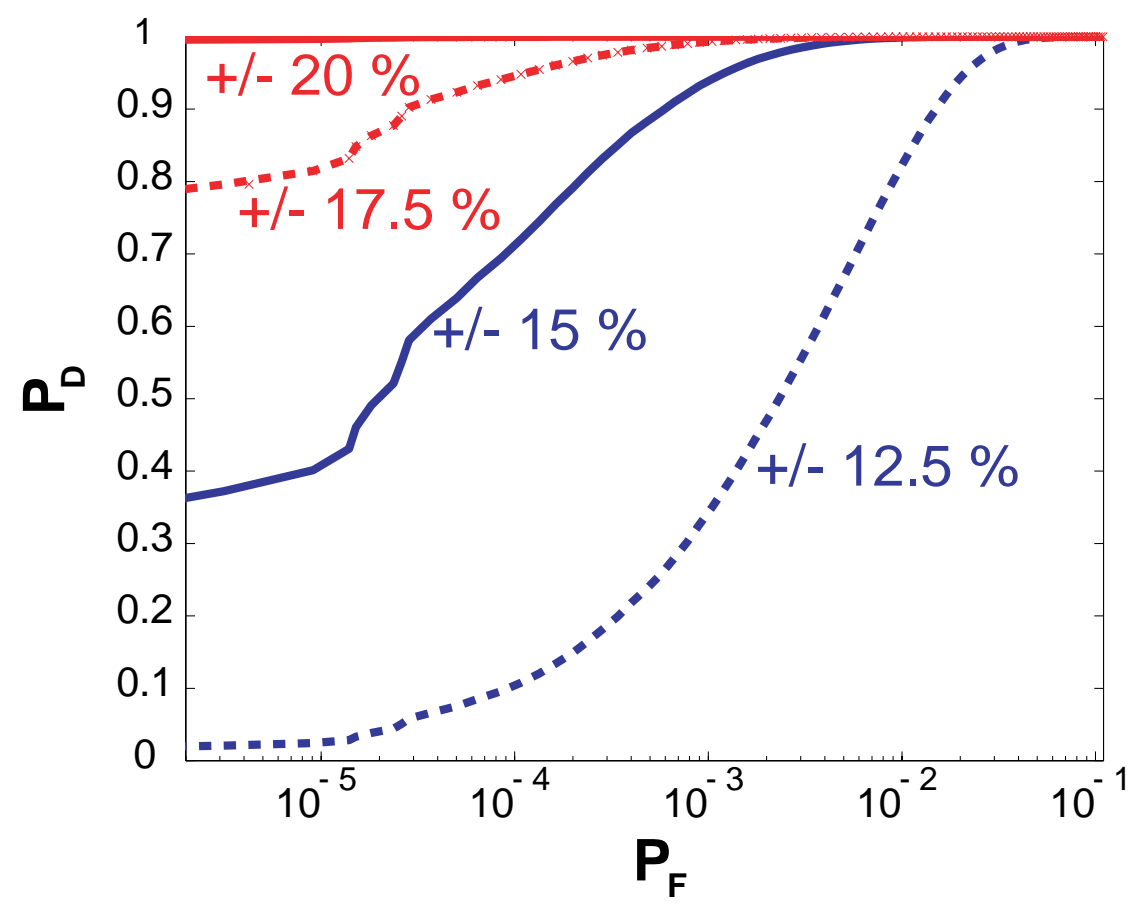

Fig. 15. Receiver Operating Characteristics of a pressure fault detector. 


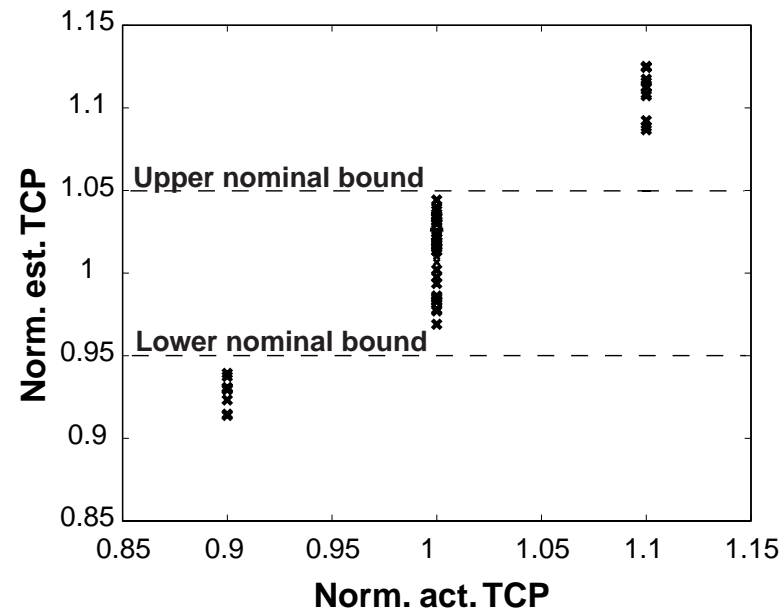

(a) TCP sensor fault detector validation result

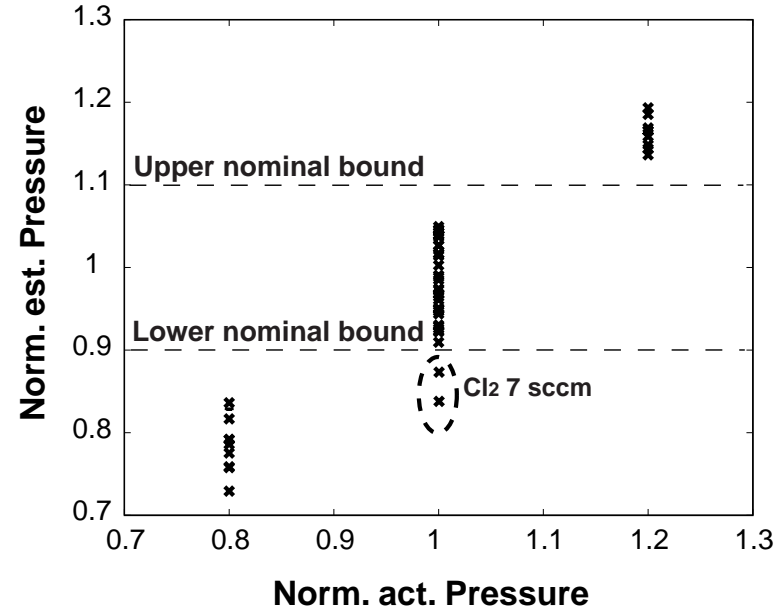

(b) Pressure sensor fault detector validation result

Fig. 16. Sensor fault detector validation experiment result. 


\begin{tabular}{l|r|r}
\hline Etch step & BT & ME \\
\hline TCP power (W) & 200 & 250 \\
BIAS power (W) & 40 & 180 \\
Pressure (mTorr) & 13 & 10 \\
$\mathrm{C}_{2} \mathrm{~F}_{6}(\mathrm{sccm})$ & 100 & 0 \\
$\mathrm{Cl}_{2}(\mathrm{sccm})$ & 0 & 15 \\
$\mathrm{HBr}(\mathrm{sccm})$ & 0 & 75 \\
\hline
\end{tabular}

TABLE I

Etch recipe used for $\mathrm{n}$-doped poly-Si in a Lam TCP 9400SE plasma etcher. 


\begin{tabular}{l|c|c|c|c|c}
\hline & TWP & BIAS & $\begin{array}{c}\text { Pressure } \\
\text { (mTorr) }\end{array}$ & $\begin{array}{c}\mathrm{Cl}_{2} \\
\text { (sccm) }\end{array}$ & $\begin{array}{c}\mathrm{HBr} \\
(\mathrm{sccm})\end{array}$ \\
\hline nominal value & 250 & 180 & 10 & 15 & 75 \\
\hline $\begin{array}{l}\text { setpoint used for } \\
\text { validation (\# of repeat) }\end{array}$ & $225(10)$ & $135(4)$ & $8(18)$ & $7(2)$ & $86(3)$ \\
\hline
\end{tabular}

TABLE II

Etch recipe used in a validation experiment. 